\title{
UMA DISCUSSÃO SOBRE A CONSTRUÇÃO DA ECONOMIA DA LONGEVIDADE EM GOIÁS E AS POLÍTICAS PÚBLICAS NACIONAIS VOLTADAS PARA OS IDOSOS
}

\section{A DISCUSSION ON THE CONSTRUCTION OF LONGEVITY ECONOMICS IN GOIAS AND PUBLIC NATIONAL POLICIES TARGETING THE ELDERLY}

\author{
Bianca Faria Loureiro $^{1}$ \\ Miguel Arruda ${ }^{2}$
}

\section{RESUMO}

O objetivo geral deste artigo é analisar a dinâmica do envelhecimento demográfico brasileiro e goiano, bem como as políticas públicas em vigor, através da apresentação do histórico do tema envelhecimento e de sua inserção na pauta das políticas públicas no Brasil, da descrição da situação do idoso brasileiro na atualidade com destaque para a necessidade de políticas públicas em vigor e da comparação do idoso no Brasil e no estado de Goiás, com relação às políticas públicas e propostas de garantia de qualidade de vida do idoso na região. Para essa análise, os dados comparativos elencados são os dos Censos de 2000 e 2010 do IBGE que circundam nos seguintes quesitos: índice de envelhecimento; índice de dependência e taxa de fecundidade. Nisso, é possível obter resultados que demonstram um fenômeno de envelhecimento acelerado e que vêm ganhando espaço na agenda das políticas sociais, mas que ainda necessitam de uma promoção de envelhecimento ativo com políticas públicas voltadas mais para a área da saúde.

Palavras-chave: Economia da longevidade. Políticas públicas. Envelhecimento populacional.

\begin{abstract}
The aim of this paper is to analyze the dynamics of demographic aging in Brazil and Goias, and public policies in place, by presenting the background of the aging theme and its inclusion in the public policies agenda in Brazil, the description of the situation of the elderly in Brazil today, highlighting the need for public policies in force and the comparison of the elderly in Brazil and in the state of Goias, in relation to public policies and proposals for life quality assurance for the elderly in the region. For this analysis, the listed comparative data were gathered from the IBGE Census of 2000 and 2010, comprising the following questions: aging index; dependency index and fertility rate. Hence, it is possible to obtain results that show an accelerated aging phenomenon that is gaining space on the agenda of social policies, but still require the promotion of active aging with more public policies towards health.
\end{abstract}

Keywords: Longevity economics. Public policies. Population aging.

\section{Introdução}

O que define o envelhecimento de uma população é o número absoluto de habitantes idosos. Segundo a Organização Mundial de Saúde (OMS, 2002), é considerado idoso o indivíduo de um país em desenvolvimento com a idade igual ou superior a 65 anos e nos países desenvolvidos com 60 anos ou mais. A Comissão Econômica para a América

\footnotetext{
${ }^{1}$ Graduanda na Universidade Federal de Goiás. Avenida Esperança, s/n - Setor Itatiaia, Goiânia - GO, 74690-900. E-mail: bianca.f.loureiro@hotmail.com

${ }^{2}$ Graduanda na Universidade Federal de Goiás. Avenida Esperança, s/n - Setor Itatiaia, Goiânia - GO, 74690-900. E-mail: miguelarruda_@hotmail.com
} 
Latina e o Caribe (CEPAL, 2003) classifica os países em quatro categorias de acordo com a intensidade do envelhecimento de cada país, o Brasil foi classificado como envelhecimento moderado avançado (a penúltima categoria de intensidade).

De acordo com relatório das Nações Unidas (ONU, 2006), até 2025 o Brasil ocupará o sexto lugar no ranking dos países com mais habitantes idosos. Além disso, outros fatores como a diminuição da fecundidade (taxa de filhos por mulher) e a expectativa de vida cada vez maior (longevidade) podem agravar problemas conjunturais brasileiros.

Diante dessa perspectiva, o objetivo geral deste artigo é analisar a dinâmica do envelhecimento demográfico brasileiro e goiano, assim como as políticas públicas em vigor. Especificamente, pretende-se: (i) apresentar o histórico do tema envelhecimento e sua inserção na pauta das políticas públicas no Brasil; (ii) descrever a situação do idoso brasileiro na atualidade com destaque para a necessidade de políticas públicas em vigor e (iii) comparar a situação do idoso no Brasil e em Goiás, políticas públicas e propostas de garantia de qualidade de vida do idoso na região.

Para atender a esses objetivos, será usada a base de dados do Instituto Brasileiro de Geografia e Estatística (IBGE), do Instituto de Pesquisa de Econômica Aplicada (IPEA) e bibliografia específica da área.

Este artigo é composto pela introdução; referencial teórico; metodologia; resultados e discussões e considerações finais.

\section{Referencial teórico}

Políticas públicas são um conjunto de ações, programas, planos e metas desenvolvidos pelo governo com o fim de alcançar o bem-estar social e satisfazer o interesse público (LOPES, 2007).

Segundo Amaral, os estágios para a criação de políticas públicas são:

- formação da agenda;

- formulação de políticas;

- escolha de ações;

- implementação; e

- avaliação. 
Dessa forma, será mostrada a inserção do tema envelhecimento populacional na agenda internacional das políticas públicas. A ONU realizou duas Assembleias Mundiais, em Viena em 1982 e em Madri em 2002. Entre a apresentação dos planos serão destacadas as políticas nacionais que entraram em vigor.

O Plano de Viena foi a primeira Assembleia Mundial nacional de políticas públicas para a população idosa. Entre os objetivos do plano estão a garantia à segurança econômica e social e a integração do idoso no processo de desenvolvimento dos países. Essa primeira assembleia mundial colocou na agenda internacional as questões relacionadas ao envelhecimento individual e da população. E ainda estabeleceu um conjunto de recomendações, visando promover a independência do idoso, o que ocasiona um aumento dos gastos públicos, especialmente na área social, como a provisão de pensão, a aposentadoria e assistência à saúde (CAMARANO, 1999).

A OMS conceitua o envelhecimento ativo como o processo que busca melhorar a qualidade de vida à medida que a população envelhece aprimorando áreas como a saúde, segurança, participação, mercado de trabalho, seguridade social e educação. O envelhecimento ativo pode ajudar a compensar o aumento dos custos com pensões, aposentarias e assistência médica e ajuda a garantir os direitos de cidadania do idoso e à igualdade de oportunidades (OMS, 2002, p. 18).

A organização defende que as políticas públicas devem dividir as responsabilidades entre os indivíduos, Estado e sociedade. No Brasil, a Lei no 8.842/1994 é reconhecida como uma das mais avançadas do mundo e baseia-se nesses princípios. A lei define que para fins legais a velhice começa a partir dos 60 anos.

A Previdência Social é um seguro social que beneficia monetariamente pessoas em situação de vulnerabilidade, cobre riscos genéricos ou específicos de eventos futuros. Tem como objetivo evitar a pobreza entre as pessoas que não podem exercer atividade laboral por motivo de saúde e garantir a reposição de renda aos seus segurados contribuintes que aposentaram (COSTA, 2007, p. 307). Para a aposentadoria compulsória em termos de Regimes Próprios Previdenciário, a idade necessária é de 70 anos. Já para a aposentadoria no Regime Geral de trabalhador rural, a idade exigida é de 55 e 60, para mulheres e homens, respectivamente. Para trabalhadores urbanos é de 65 e 60, para homens e mulheres, respectivamente (COSTA, 2007, p. 308). 
A Lei Orgânica de Assistência Social determina que a assistência social é um direito do cidadão necessitado e dever do Estado provê-la. O Benefício de Prestação Continuada (BPC) é regulamentado pela Lei Orgânica de Assistência Social (LOAS). Esse benefício concede um salário mínimo à pessoa com 65 anos ou mais cuja renda per capita seja inferior a um quarto de salário mínimo vigente. Em 2012, o BPC tinha 3,6 milhões de beneficiários em todo o país, dos quais 1,7 milhão eram pessoas idosas.

Em 2002, foi realizada em Madri a Segunda Assembleia Mundial com o tema de envelhecimento populacional. Nessa assembleia foi aprovado um novo plano de ação que deverá orientar a adoção de medidas sobre o envelhecimento no início do século XXI. Nesse plano foi ressaltada a necessidade da ação colaborativa entre o Estado e a sociedade. O Plano de Madri fundamenta-se em três princípios:

- o envelhecimento ativo;

- envelhecimento saudável (saúde e bem-estar); e

- criação de um ambiente propício e favorável ao envelhecimento.

Em 2003, entrou em vigor a Lei n ${ }^{\circ} 10.741$, que aprova o Estatuto do Idoso, uma das principais leis de proteção ao direito do idoso. Essa lei garante o direito à igualdade dos idosos e foi uma adequação às orientações do Plano de Madri (FERNANDES; SOARES, 2012, p. 1497).

Em 2006, a Portaria GM no 2.528 criou a Política Nacional de Saúde da Pessoa Idosa (PNSPI) que define a atenção à saúde da população idosa e terá como porta de entrada a Atenção Básica/Saúde da Família, tendo como referência a rede de serviços especializada de média e alta complexidades.

Foi estabelecido, então, o Pacto pela Saúde do SUS, e a saúde do idoso torna-se uma das seis prioridades pactuadas entre as três esferas de governo no SUS (Portaria GM/MS 399/2006).

Em 2006, acontece a primeira Conferência Nacional dos Direitos da Pessoa Idosa, na qual foram aprovadas deliberações para ampliar os direitos dos idosos e construir a Rede Nacional da Pessoa Idoso (Renadi) (FERNANDES; SOARES, 2012, p. 1497).

Os países devem criar programas e ações para a integração do idoso no processo produtivo, bem como reconhecer que um número maior de idosos na população não 
significa um "ônus demográfico", causador do esgotamento de recursos sociais. Um número maior de idosos deve ser encarado como uma acumulação de capital humano, social e econômico, elementos necessários para a manutenção do bem-estar da sociedade.

Hoje o Brasil está passando por uma transição demográfica de um regime de grande crescimento populacional para uma fase de crescimento moderado, que pode chegarm inclusive, a chegar, daqui algumas décadas, a uma redução de sua população. Em 1965, a fecundidade começa a cair no país, chegando, em 2005, ao que chamam de taxa de reposição demográfica (a média de 2,1 filhos por mulher). Nesse panorama, a tendência é de diminuição da fecundidade, e a consequência é o decrescimento populacional. Segundo as projeções da ONU, até 2040, a população brasileira vai diminuir graças ao envelhecimento populacional e à baixa fecundidade (ALVES, 2010, p. 9-10). A expectativa de vida ao nascer deve aumentar de 71 anos para 79,9 anos. A população com mais de 65 anos representará cerca de $22,5 \%$ da população.

Essa população demandará uma gama de serviços existentes ou insuficientes, como a necessidade de medicina especializada para diagnóstico e tratamento de doenças típicas de idade avançada e prevenção de doenças crônicas. Esse cenário acarretará em um peso relativamente grande e crescente nas contas públicas (ALVES, 2010, p. 31).

Outra preocupação com o envelhecimento populacional e a longevidade é a sustentabilidade do sistema previdenciário público e privado, já que uma população idosa terá um impacto considerável sobre a População Economicamente Ativa (PEA), que compreende pessoas de 15 a 65 anos (ALVES, 2010, p. 31).

\section{Metodologia}

O objetivo geral deste artigo é analisar a dinâmica do envelhecimento demográfico brasileiro e goiano, assim como as políticas públicas em vigor.

Para atender a esse objetivo a análise será dividida em duas partes. A primeira parte consiste no estudo histórico das políticas públicas existentes desde o Plano de Viena e descrever a situação do idoso. 
Os dados comparativos elencados para comparar e descrever a necessidade dessas políticas e sua ampliação são os do Censos de 2000 e 2010 do IBGE. Os seguintes quesitos serão avaliados:

- Índice de Envelhecimento: relação entre a população idosa (a partir de 65 anos) e população jovem (com menos de 15 anos).

- Índice de Dependência: porcentagem de idosos em relação ao número de pessoas ativas.

- Taxa de Fecundidade: relação entre os nascimentos e o número de mulheres entre 15 e 49 anos completos.

Para complementar essa análise e demonstrar a situação do idoso serão usadas as Sínteses dos Indicadores Sociais publicados pelo IBGE nos anos de 2008 e 2011.

\section{Resultados e discussões}

Como foi destacado neste artigo, o fenômeno do envelhecimento da população brasileira vem ocorrendo de forma acelerada e ganhando espaço na agenda das políticas sociais, por isso há a necessidade de políticas públicas de saúde e de promoção do envelhecimento ativo.

\subsection{Envelhecimento populacional no Brasil}

Comparando as pirâmides etárias a seguir, nota-se a diminuição da base da pirâmide e o seu alongamento (devido ao aumento da expectativa de vida). Além disso, é possível perceber que a maior parte da população concentra-se na fase adulta.

Figura 1 - Pirâmide etária do Brasil em 2000

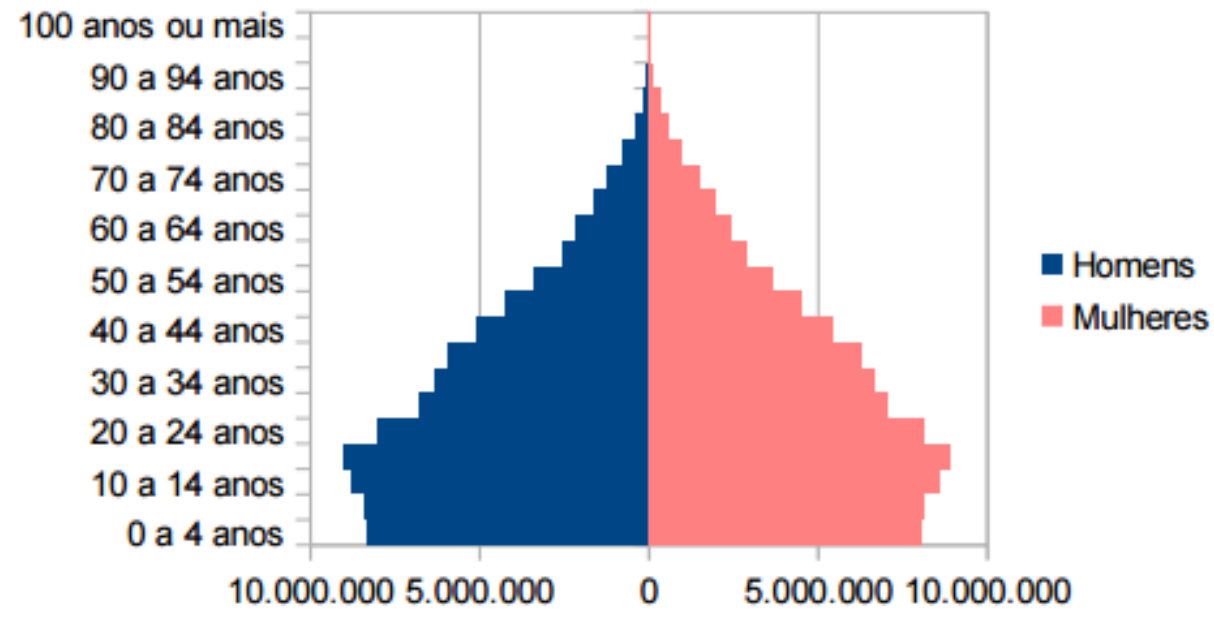

Fonte: elaborada pelos autores (2014). 
Figura 2 - Pirâmide etária do Brasil em 2010

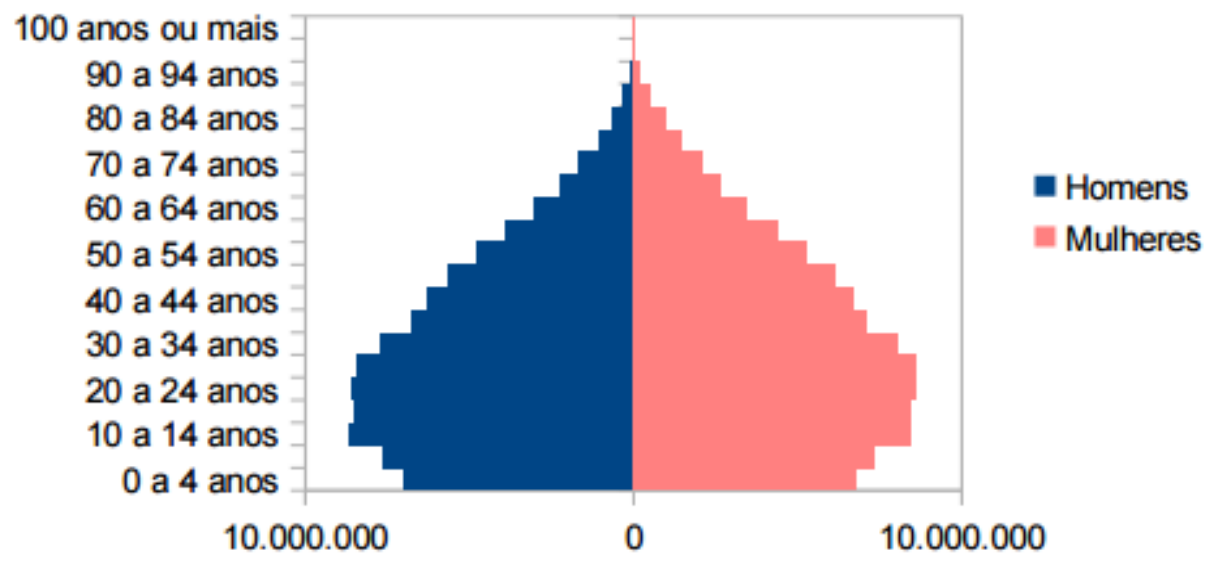

Fonte: elaborada pelos autores (2014).

Analisando a base da pirâmide etária brasileira, em 2000, o número de pessoas de 0 a 14 anos era de 50.316.181. Em 2010, a população com a mesma faixa etária somava 45.941.636, portanto houve um decréscimo populacional de $8,69 \%$, essa retração pode ser explicada através da Tabela 1 que mostra a queda da taxa de fecundidade entre os Censos de 2000 e 2010, que passou de 2,38 para 1,9.

O corpo da pirâmide é representado pela População Economicamente Ativa que aumentou 19,25\% entre os dois censos, passando de 109.629.647 para 130.728.559.

O topo da pirâmide etária é composto pelas pessoas com mais de 65 anos. De 2000 a 2010, o número de idosos passou de 9.927 .028 para 14.085 .605 , o que representa um crescimento de 41,89\%. Com isso, o índice de envelhecimento populacional que era de 19,73\%, em 2000, passou para 30,66\%, em 2010. E o grau de dependência subiu de $7,99 \%$ para $12,85 \%$.

Tabela 1 - Índices do Brasil em 2000 e 2010

\begin{tabular}{lrr}
\hline & 2000 & 2010 \\
\hline Indice de Envelhecimento & $19,73 \%$ & $30,66 \%$ \\
Indice de dependência & $7,99 \%$ & $12,85 \%$ \\
Taxa de Fecundidade & 2,38 & 1,9 \\
\hline
\end{tabular}

Fonte: dados do IBGE, Censo 2000 e 2010.

De acordo com a Síntese dos Indicadores Sociais publicada em 2008, a maioria da população idosa do Brasil é composta por mulheres. As mulheres apresentam maior longevidade. 
A educação é um aspecto preponderante para determinar o bem-estar durante a velhice, pois o nível de educação influencia a renda, tipo de trabalho, aposentadoria, lazer, convivência familiar e saúde. Os idosos têm o nível de escolaridade baixa, a média é de 3,3 anos de estudo. Entre os idosos com mais de 80 anos a média é de 2,4 anos de estudo. E 32,2\% do total de idosos têm menos de um ano de estudo. Os homens idosos têm mais condição de alfabetização $(68,9 \%)$, enquanto as mulheres idosas são 69,7\%. Camarano e Pasinato (2004) destacam que apenas 5,54\% dos idosos têm mais de 12 anos de estudo, os quais têm uma renda $412 \%$ superior à da maior parte da população idosa.

Segundo Felix (2009), a maior parte da renda dos idosos é proveniente da Seguridade Social. Em 2007, 84\% dos idosos recebiam aposentaria ou pensão. Na área rural, a quantidade de idosos que recebem algum tipo de benefício é de $88 \%$. Cerca de $22,5 \%$ dos idosos com mais de 65 anos continuam trabalhando para complementar a renda advinda da aposentadoria.

Conforme o IBGE (2008), o rendimento domiciliar per capita é um importante indicador do nível de bem-estar de uma população. Em 2007, poderiam ser considerados em situação de pobreza cerca de 12,6\% dos idosos do Brasil.

Analisando a série de dados das PNADs dos anos de 1997, 2002 e 2007, nota-se uma queda significativa na quantidade de idosos em situação de pobreza ao longo do período. Esse cenário, provavelmente, é uma consequência da Lei Orgânica da Assistência Social (LOAS), que destinava um salário mínimo a idosos com 70 anos ou mais e com renda per capita de um quarto de salário mínimo vigente em 1998. Mais tarde, a idade mínima necessária para requerer o benefício foi reduzida para 67 anos, e, em 2004, para 65 anos de idade.

Conforme a Síntese dos Indicadores Sociais divulgada em 2011, a maioria dos idosos recebe algum benefício da Previdência Social. Um em cada quatro idosos vive com renda mensal per capita inferior a um salário mínimo. Felix (2009) ressalta que esse sistema de benefício previdenciário contributivo e outras ações como o Bolsa Família são responsáveis pela redução da desigualdade de renda no país. 
Essa análise da condição de vida do brasileiro feita pelo IBGE (2008) destaca ainda que a contribuição dos idosos na renda familiar chega a 53\%, mais da metade do total da renda domiciliar. Segundo Felix (2009):

No Brasil os arranjos familiares têm significado um importante suporte para as gerações mais novas devido à renda dos idosos. No entanto, há inúmeras interrogações sobre os benefícios para os idosos e se estes, em fase de dependência, estão realmente usufruindo de cuidados básicos. (FELIX, 2009, p. 52)

O mesmo autor ainda destaca a necessidade de ampliar a política de atendimento ao idoso no Brasil. Em 2005, o Ministério de Desenvolvimento Social financiou 1.146 instituições de cuidado de longa permanência com cerca de 24.859 idosos. Essa política não atendeu nem $0,2 \%$ do contingente de brasileiros idosos.

Em 1970, as políticas públicas da área de saúde eram voltadas para o planejamento familiar como consequência do acelerado crescimento populacional. Dentre o conjunto dessas políticas, a constituição do Sistema Único de Saúde (SUS) com a atenção primária destinada à saúde materna e infantil contribuiu para o aumento da expectativa de vida e a redução das taxas de mortalidade (MENDES, 2012, p. 955).

Hoje, na pauta das políticas públicas de saúde está o crescimento da população ativa e idosa. Esse novo perfil demográfico demanda uma maior quantidade de atendimentos de urgências e emergências por traumatismo, acidentes vasculares cerebrais e condições cardiológicas. Nas idades mais avançadas, os tratamentos médicos tornam-se constantes e mais onerosos (MENDES, 2012, p. 962).

Em projeções realizadas levando-se em consideração o envelhecimento da população e a variação do nível da renda do país até meados do século XXI (tendo em conta um crescimento médio do rendimento per capita de $2,5 \%$ ao ano), a demanda por serviços de saúde para consultas médicas deverá crescer 59\%, para exames, 96\% para tratamentos, $122 \%$, e para internações, $39 \%$. Em relação ao gasto com saúde, o envelhecimento da população deverá acarretar um aumento do gasto com saúde em relação ao PIB de aproximadamente $30 \%$ até 2050 , dos atuais $8,2 \%$ para $10,7 \%$. (MENDES, 2012, p. 961)

De acordo com a PNSPI, o principal problema que pode afetar a vida durante a velhice é a perda da capacidade funcional. Diante do exposto, família, Estado e sociedade devem se comprometer a implantar uma vida digna para todos independentemente da idade. Aprender a envelhecer é uma necessidade social, assim, 
deve ser promovida a educação gerontológica por toda a vida. Só a educação pode ser o agente transformador da vida coletiva e individual da pessoa idosa.

Figura 3 - Pirâmide etária de Goiás em 2000

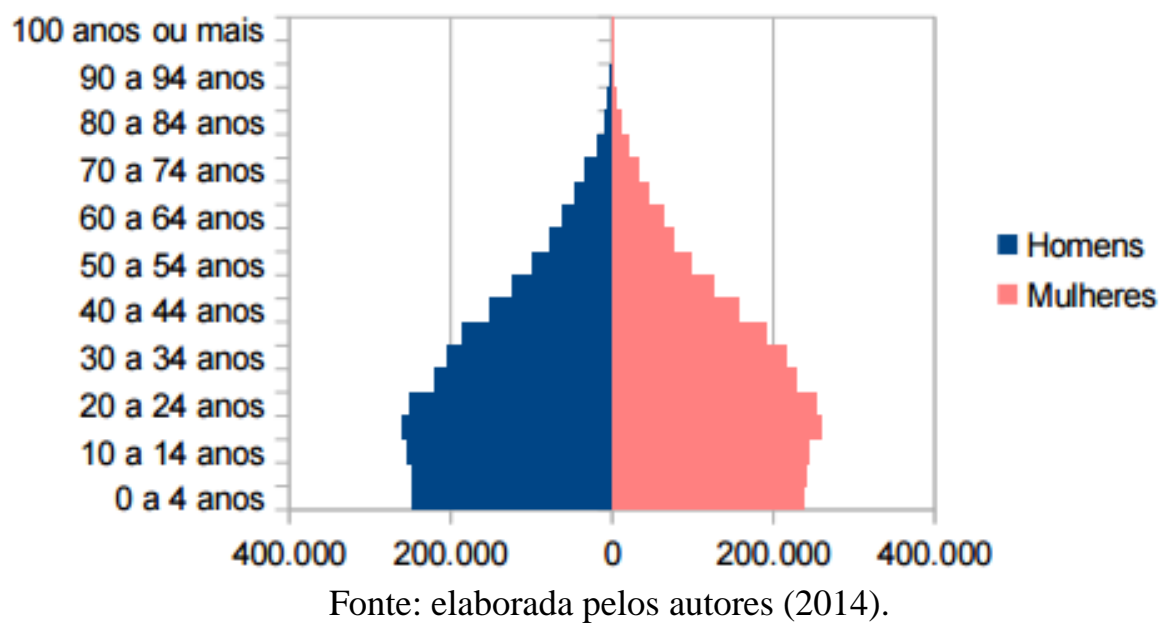

Figura 4 - Pirâmide etária de Goiás em 2010

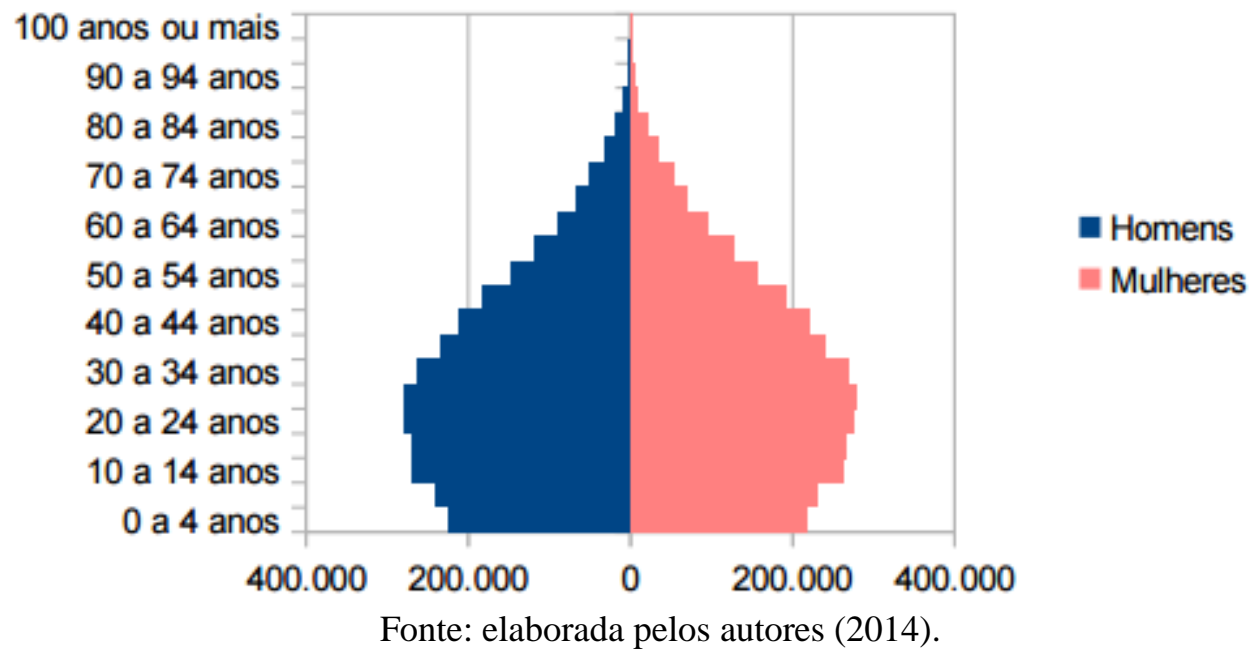

No estado de Goiás, o número absoluto de pessoas de 0 a 14 anos era de 1.446.985. Em 2010, essa mesma faixa da população era de 1.442 .406 , portanto houve um decréscimo populacional de 1,68\%. É possível explicar essa retração através da Tabela 2, que mostra a queda da taxa de fecundidade entre os Censos de 2000 e 2010, o número de filhos por mulher passou de 2,24 para 1,86.

A População Economicamente Ativa (pessoas entre 15 e 64 anos) aumentou 26,70\% entre os dois períodos em análise, ou seja, passou de 3.304.928 para 4.187.402.

O número absoluto de idosos, entre 2000 e 2010, passou de 358.309 para 373.980, o que representa um crescimento de 4,37\%. O índice de envelhecimento 
populacional, que era de $15,83 \%$ em 2000 , passou para $25,93 \%$ em 2010 . E o grau de dependência, de $7,03 \%$ para $8,93 \%$.

Segundo a Pesquisa Nacional por Amostra de Domicílios (PNAD), em 2011, os idosos em Goiás correspondiam a 8,24\% da população do estado. De acordo com o Instituto Mauro Borges, a quantidade de idosos subiu 85\% desde 2000, superando a população de crianças até 4 anos.

Tabela 2 - Índices de Goiás em 2000 e 2010

\begin{tabular}{lrr}
\hline & 2000 & 2010 \\
\hline taxa de fecundidade & 2,24 & 1,86 \\
Índice de envelhecimento & $15,83 \%$ & $25,93 \%$ \\
Índice de dependência & $7,03 \%$ & $8,93 \%$ \\
\hline
\end{tabular}

Fonte: dados do IBGE, Censo de 2000 e 2010.

De acordo com Soares e colaboradores (2002) alguns aspectos relevantes da situação do idoso no estado de Goiás:

- de 2461 idosos entrevistados, 62,4\% consideravam a própria saúde ruim e $66,6 \%$ foram internados no último ano; além disso, 51,2\% dos idosos informaram que moravam distantes dos serviços de saúde;

- as internações de pessoas acima de 65 anos registradas entre o período de 1998 a 2002 foram causadas por doenças como insuficiência cardíaca congestiva, hipertensão arterial primária, doenças pulmonares obstrutivas crônicas e pneumonias;

- $61 \%$ dos idosos se declaram analfabetos; e

- $78,2 \%$ deles moravam com seus familiares, e 19,3\% sozinhos.

Em Goiás, o projeto de assistência ao idoso é dividido em três partes: asilar, conviver e domiciliar. Esses projetos são responsabilidade da Secretaria de Cidadania e Trabalho, através da Superintendência de Assistência Social e do Idoso.

O projeto Conviver é realizado em Goiânia no Jardim Novo Mundo e tem 600 idosos cadastrados no centro de convivência. Além disso, na Vila Mutirão, o projeto mantém moradia para 60 idosos. Ao todo, a Superintendência dá assistência a 12 mil idosos espalhados em 187 municípios de Goiás. O Conselho Estadual do Idoso (CEI) tem o objetivo de promover as políticas de proteção dos direitos dos idosos. 
Em 2007, o estado de Goiás deu início à Política de Atenção à Saúde da Pessoa Idosa para tentar proporcionar ao idoso o acesso às ações de promoção à saúde, prevenção de doenças, recuperação e reabilitação, com o intuito de propagar o envelhecimento saudável e ativo. Para implantar essa política foram escolhidos três eixos norteadores: Organização e Estruturação da Rede na Atenção à Saúde da Pessoa Idosa, Capacitação de Recursos Humanos e Gestão da Informação.

Tanto estado de Goiás, como o Brasil todo estão em processo de envelhecimento populacional, caracterizado por queda na taxa de fecundidade e aumento da taxa de longevidade.

\section{Considerações finais}

O envelhecimento da população brasileira vem aumentando ano a ano e há uma necessidade de políticas públicas voltadas para a saúde e a promoção do envelhecimento ativo.

Portanto, o objetivo geral deste artigo foi analisar a dinâmica do envelhecimento demográfico brasileiro e goiano, assim como as políticas públicas em vigor.

Após realizar um apanhado geral das políticas públicas no Brasil e em Goiás e dos poucos estudos existentes voltados para essa temática, este trabalho concluiu que há a necessidade da ampliação da pesquisa e investigação dos impactos econômicos causados pelo envelhecimento populacional. Nesse sentido, a construção da Economia da Longevidade é a chave para conglomerar os estudos pertinentes nas áreas da Economia, como: Economia da Saúde, Economia do Setor Público (avaliar os impactos e a criação de novas políticas públicas), Economia do Seguro, Economia Regional e Urbana (para adaptação de espaços e melhorias de serviços) e Demografia.

É preciso mudar a visão midiática imposta do idoso como um "peso" para a economia do país, já que os gastos com serviços públicos são mais onerosos e constantes e essa parcela da população tornou-se "improdutiva". A formação da Economia da Longevidade depende também de uma maior participação social dos idosos nas decisões do Estado, para cobrar e requisitar serviços públicos e leis que os protejam. O processo de envelhecimento é comum a todos, e a garantia desse direito é de responsabilidade do Estado, de cada indivíduo e da sociedade. 


\section{REFERÊNCIAS}

ALVES, V.P.; VIANNA, L.G. Políticas públicas para a educação gerontológica na perspectiva da inserção social do idoso: desafios e possibilidades. Ensaio: aval. pol. públ. Educ., v. 18, n. 68, p. 489-510, 2010.

BRASIL. Decreto nº 1.948, de 3 de julho de 1996

Lei $n^{\circ} 10.741$, de $1^{\circ}$ de outubro de 2003. Dispõe sobre o Estatuto do Idoso e dá outras providências. Diário Oficial da União, 3 out. 2003.

Portaria do Gabinete do Ministro de Estado da Saúde n 1395, de 9 de dezembro de 1999. Aprova a Política Nacional de Saúde do Idoso e dá outras providências. Diário Oficial da União, 9 dez. 1999.

Portaria $\mathrm{n}^{\mathrm{o}}$ 2.528, de 19 de outubro de 2006. Aprova a Política Nacional de Saúde da Pessoa Idosa e determina outras providências. Diário Oficial da União, 19 out. 2006.

Portaria no 399/GM, de 22 de fevereiro de 2006.

. Regulamenta a Lei ${ }^{\circ} 8.842$, de 4 de janeiro de 1994, que dispõe sobre a Política Nacional do Idoso, e dá outras providências. Diário Oficial da União, 4 jul. 1996.

Lei $\mathrm{n}^{\circ}$ 8.842, de 4 de janeiro de 1994. Dispõe sobre a Política Nacional do Idoso, cria o Conselho Nacional do Idoso e dá outras providências. Diário Oficial da União, 4 jan. 1994,

CAMARANO, A.A.; PASINATO, M.T. O envelhecimento populacional na agenda das políticas públicas. In: CAMARANO, A.A. (Org.). Os novos idosos brasileiros: muito além dos 60? Rio de Janeiro: Ipea, 2004. p. 253-292.

FELIX, J. Economia da longevidade: o envelhecimento da população brasileira e as políticas públicas para os idosos. Dissertação de mestrado em Economia Política, São Paulo, PUC-SP, 2009.

FERNANDES, M. T. O.; SOARES, S. M. O desenvolvimento de políticas públicas de atenção ao idoso no Brasil. Revista da Escola de Enfermagem da USP, v. 46, n. 6, p. 1494-1502, 2012. 
IBGE. Síntese dos Indicadores Sociais. Rio de Janeiro: IBGE, 2008.

. Síntese dos Indicadores Sociais. Rio de Janeiro; IBGE, 2011.

LIMA-COSTA, M.F.; LOYOLA FILHO, I.A.; MATOS, D.L. Tendências nas condições de saúde e uso de serviços de saúde entre idosos brasileiros: um estudo baseado na pesquisa nacional por amostra de domicílios (1998, 2003). Cad. Saúde Pública, v. 23, n. 10, p. 2467-2478, 2007.

LOPES, R. G. C. Saúde na velhice: as interpretações sociais e os reflexos no uso do medicamento. São Paulo: Educa, 2000.

ONU. Source: Population Division of the Department of Economic and Social Affairs of the United Nations Secretariat, World Population Prospects: The 2006 Revision and World Urbanization Prospects: The 2005 Revision. Disponível em: <http://esa.un.org/unpp>. Acesso em: 22 set. 2014.

PÁDUA, A. A. S.; COSTA, E. R. Políticas Públicas de Previdência e Assistência Social ao Idoso. Estudos, v. 34, n. 5/6, p. 305-317, maio/jun. 2007.

SOARES, E. et al. Diagnóstico da situação da população idosa em quatorze municípios do estado de Goiás. Goiânia: UFG, 2002. 46p.

WHO. Active Ageing - A Police Framework. A Contribution of the World Health Organization to the second United Nations World Assembly on Aging. Madrid, Spain, April, 2002.

Recebimento dos originais: $11 / 11 / 2013$

Aceitação para publicação: 22/05/2014 